\title{
Effect of Assembly Condition on Dynamic Performance of C/C Composite Finger Seal
}

\author{
WANG Li'na ${ }^{1, a^{*}, \text { CHEN Guoding }}{ }^{1, b}$, SU Hua ${ }^{1}$, \\ YANG Guangmei ${ }^{1}$, ZHANG Yanchao ${ }^{2}$ \\ ${ }^{1}$ School of Mechanical Engineering, Northwestern Polytechnical University, Xi'an, 710072, China \\ ${ }^{2}$ Xi'an University of Technology, Xi'an, 710048, China \\ a wangxiweigood@163.com, b gdchen@nwpu.edu.cn
}

Keywords: finger seal; aeroengine; equivalent dynamic model; $\mathrm{C} / \mathrm{C}$ composite

Abstract. The performance of finger seal as an important component has decisive influence on performance of aeroengine. The complicated operating state of aeroengine, such as assembly condition, also has important influence on finger seal performance. However, equivalent dynamic model has not considered this factor, the effect of that on calculation accuracy of finger seal performance analysis is inevitable. Aiming at the problems mentioned above, a distributed mass is proposed in this paper, and the influence of assembly condition on finger seal performance is investigated. It is shown that, assembly condition of finger seal has important influence on dynamic performance of finger seal. The present work is more close to actual engineering, and the work may have certain academic and practical values.

\section{Introduction}

Finger seal as a kind of flexible seal has a better performance-price ratio, it is generally regarded as a seal device with potential application prospects in secondary air systems and bearing chambers in modern aeroengines. Therefore, it has been drawn more and more attentions and studied recently ${ }^{[1,2]}$.

Arora et al. ${ }^{[3]}$ proposed a finger seal structure that had pressure balance chamber, which could decrease friction between finger element and aft cover plate. Their following experiment work also proved the advantage of this structure, namely, the leakage of finger seal was decreased ${ }^{[4]}$. Gibson et al. proposed a new combined stiffness of finger stick in the finger element along axial direction, in order to decrease the leakage of finger seal ${ }^{[5]}$. Chen et al. analysed the dynamic performance of 2.5 dimension $\mathrm{C} / \mathrm{C}$ composite finger seal with the equivalent dynamic model of distributed mass, and then compared that with Co-based alloy finger seal. It is shown that the contact pressure of $\mathrm{C} / \mathrm{C}$ composite finger seal is less than that of Co-based alloy, however its leakage rate is larger ${ }^{[6,7]}$.

The complicated operating state of aeroengines, such as assembly condition, also has important influence on finger seal performance. However, so far the effect of assembly condition on dynamic performance of finger seal has not been investigated. It is necessary to make up this limitations of previous work, and the dynamic performance analysis of finger seal is carried out considering assembly condition.

A distributed mass equivalent dynamic model is proposed, and the influence of assembly condition on finger seal performance is investigated. The current work in this paper is more suitable to engineering practice, and may have much academic significance and engineering values.

\section{Equivalent dynamic model of finger seal}

\section{Equivalent dynamic model of finger seal}

The mechanical behavior between the rotor and multilayer overlay finger seal can be expressed by the distributed mass equivalent dynamic model that is presented in Fig.1. In Fig.1, $m_{i}$ and $k_{i}$ are equivalent mass and equivalent structural stiffness coefficient of a single finger stick of the $i$ th finger laminate, respectively. $F_{\mathrm{fi} i}$ and $F_{\mathrm{fi} i}^{\prime}$ are frictional resistances of the neighboring finger elements (or the aft 
cover plate) on the $i$ th finger element. $x_{i}$ is displacement response of finger stick of the $i$ th finger element. As shown in Fig.1(a), the finger stick and the rotor are in the contact state, $k_{\mathrm{c}}$ is the contact stiffness coefficient between the finger stick and the rotor, and $y$ is the displacement excitation of the rotor. As shown in Fig.1(b), the finger sticks and the rotor are out of the contact state, and the fluid pressure appears between the finger sticks and the rotor due to the leakage clearance generated between them. $G_{i}$ is the fluid pressure acting on the finger sticks of the $i$ th finger element. As shown in Figs.1(a) and 1(b), the finger seal moves with the damped forced vibration.

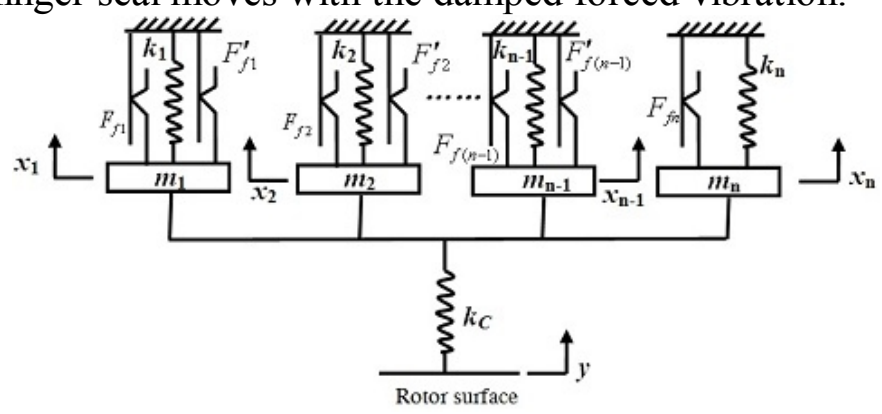

(a) Finger element and the rotor are in contact

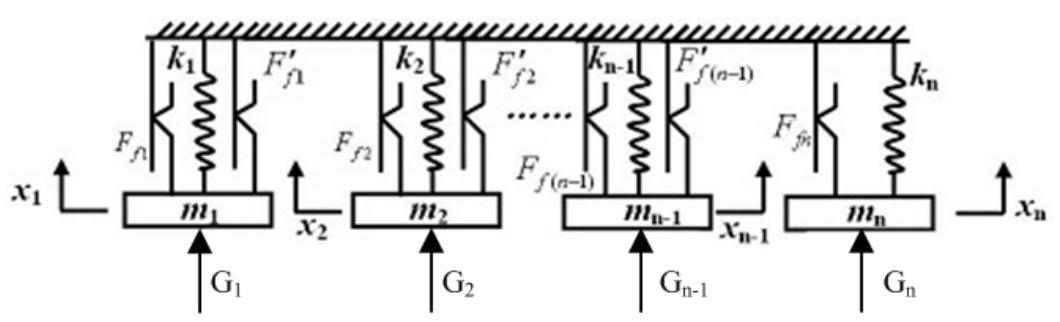

(b) Finger element and the rotor are out of contact

Fig.1 Equivalent dynamic model of finger seal based on distributed mass

When finger sticks and rotor are in contact state, the damped forced vibration differential equations of finger seal are defined as

$$
\left\{\begin{array}{l}
m_{1}=-k_{1} x_{1}+\frac{k_{c}}{n}\left(y-x_{1}\right)+F_{f 1}^{\prime}-F_{f 1} \\
m_{2}=-k_{2} x_{2}+\frac{k_{c}}{n}\left(y-x_{2}\right)+F_{f 2}^{\prime}-F_{f 2} \\
M \\
m_{n-1}=-k_{n-1} x_{n-1}+\frac{k_{c}}{n}\left(y-x_{n-1}\right)+F_{f(n-1)}^{\prime}-F_{f(n-1)} \\
m_{n}=-k_{n} x_{n}+\frac{k_{c}}{n}\left(y-x_{n}\right)-F_{f n}
\end{array}(n \geq 2 \text { and } n \text { is an integer })\right.
$$

When finger sticks and the rotor are out of contact state, the damped forced vibration differential equations of finger seal are defined as

$$
\left\{\begin{array}{l}
m_{1}=-k_{1} x_{1}+G_{1}+F_{f 1}^{\prime}-F_{f 1} \\
m_{2}=-k_{2} x_{2}+G_{2}+F_{f 2}^{\prime}-F_{f 2} \\
\mathrm{M} \\
m_{n-1}=-k_{n-1} x_{n-1}+G_{n-1}+F_{f(n-1)}^{\prime}-F_{f(n-1)} \\
m_{n}=-k_{n} x_{n}+G_{n}-F_{f n}
\end{array} \quad(n \geq 2, \text { and } n \text { is an integer })\right.
$$

where subscript $n$ is the number of finger elements. The mechanical relationship between $F_{\mathrm{fi}}$ and $F^{\prime}{ }_{\mathrm{f} i}$ is defined as

$$
F_{f i}=F_{f(i-1)}^{\prime} \quad(2 \leq i \leq n, \text { and } n \text { is an integer })
$$

\section{Treatment of equivalent parameters}

Equivalent parameters of equivalent dynamic model are confirmed based on the structure and 
working condition of finger seal:

(1) Equivalent mass

Single finger stick of finger element is equivalent to a lumped mass by the distributed mass equivalent dynamic model. Based on the conservation principle of kinetic energy, the kinetic energy of the finger stick and the finger foot are equal to that of the equivalent mass. Then the equivalent mass $m_{i}$ can be solved with Eq.(4) and presented as ${ }^{[8]}$

$$
m_{i}=m_{f}+\int_{0}^{l}(\rho h \delta) x^{2}(z) \mathrm{d} z
$$

where $m_{f}$ is the mass of finger foot, $l$ is the length of the finger stick, $\rho$ is the density of $\mathrm{C} / \mathrm{C}$ composite, $h$ is the width of finger stick, and $\delta$ is the thickness of finger element, $x(z)$ is static displacement of any point mass in finger stick.

(2) Stiffness coefficient of equivalent structure

Applying a radial load on the finger foot, and then calculating the displacement of the forced finger foot by FEM. The ratio of radial load to displacement can be confirmed as the equivalent structure stiffness coefficient.

$$
k=\frac{\Delta F}{\Delta x}
$$

(3) Contact stiffness coefficient of finger stick and rotor

The contact stiffness formula of finger stick and rotor is defined as

$$
k_{c}=\frac{A_{f}}{A_{a}} \times \frac{k_{s} k_{R}}{k_{s}-k_{R}}
$$

where $A_{\mathrm{f}}$ is the contact area between the finger stick and the rotor; $A_{\mathrm{a}}$ is the nominal contact area of the object involving the rough surface; $k_{\mathrm{s}}$ is the stiffness coefficient of no-interface block structure; $k_{\mathrm{R}}$ is the stiffness coefficient of the object involving the rough surface.

(4) Frictional resistance of finger seal system

The friction resistance between the aft cover plate and the first layer finger element and the friction resistance between the $i$ th layer finger element and the $(i+1)$ th layer finger element are consistently expressed as

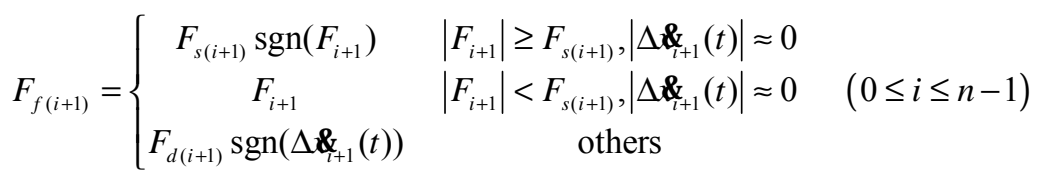

where $\Delta \alpha_{i+1}$ is the velocity difference between the $i$ th layer finger element and the $(i+1)$ th layer finger element; while $i=0, \Delta \&(t)$ is the velocity difference between the aft cover plate and the first layer finger element; $F_{\mathrm{s}(i+1)}$ and $F_{\mathrm{d}(i+1)}$ are the static friction and the dynamic friction between the aft cover plate and the first layer finger element (while $i=0$ ), or the $i$ th layer finger element and the $(i+1)$ th layer finger element, respectively.

\section{Displacement excitation of the rotor}

Combined assembly condition of finger seal with effect of unbalanced mass on the rotor displacement excitation, the rotor displacement excitation is shown as

$$
y_{i}=\Delta r \sin (\omega t) \pm d
$$

where $\Delta r$ is the radial displacement amplitude of the rotor; $\omega$ is the angular velocity of the rotor; $t$ is the time; $d$ is magnitude of interference or clearance, ' + ' and '-' express interference fit and clearance fit respectively.

\section{Fluid pressure between finger stick and the rotor}

When a clearance is generated between the finger elements and the rotor, the fluid pressure acting on finger stick in each finger element is only related to the axial position of the finger element and the pressure differential of the sealing system. If the fluid pressure is assumed to be linearly distributed along the axial direction, the fluid pressure acting on finger stick in the $i$ th finger laminate is expressed 


$$
G_{i}=P_{\mathrm{L}}+\frac{i-1 / 2}{n} \Delta P
$$

where $P_{\mathrm{L}}$ is the fluid pressure downstream from the seal and $\Delta P$ is the fluid pressure differential of the sealing system.

\section{Dynamic performance of finger seal system}

According to the average leakage gap of finger seal, the leakage rate $Q$ during one motion cycle is obtained and shown as

$$
Q=\rho_{a} \frac{\pi D_{r} \bar{h}^{3} \Delta P}{12 \eta L}
$$

where $\rho_{\mathrm{a}}$ and $\eta$ are the density and dynamic viscosity of the fluid, respectively; $L$ is seal leakage length and $D_{r}$ is the diameter of the rotor.

During one motion cycle of the rotor, the average contact pressure between the finger stick and the rotor in $i$ th finger element is shown as

$$
\bar{P}_{i}=\frac{1}{f} \sum_{j=1}^{f} \frac{F_{i}\left(t_{0}+j \Delta t\right)}{A_{i}}
$$

where $A_{i}$ is the contact area between the $i$ th layer finger laminate and the rotor; $F_{i}(t)$ is the contact force between the $i$ th layer finger laminate and the rotor; $t_{0}$ is initial time point; $f$ is the number of time steps in a running period of rotor; $\Delta t$ is the scale of time step.

\section{Results and discussion}

Dynamic performance of 2.5 dimension $\mathrm{C} / \mathrm{C}$ composite finger seal considering assembly condition is mainly studied, the analysis of finger seal is composed of nine layer finger elements by calculation limitation.

The object of single finger stick in 5th finger element is studied in this section. Effect of assembly condition on displacement is shown in Fig.2. It is shown that, in accordance with clearance fit, no clearance fit and interference fit, the displacement response of finger stick would be decreased. Corresponding to that the leakage between finger stick and the rotor would be also decreased, as shown in Fig.3.

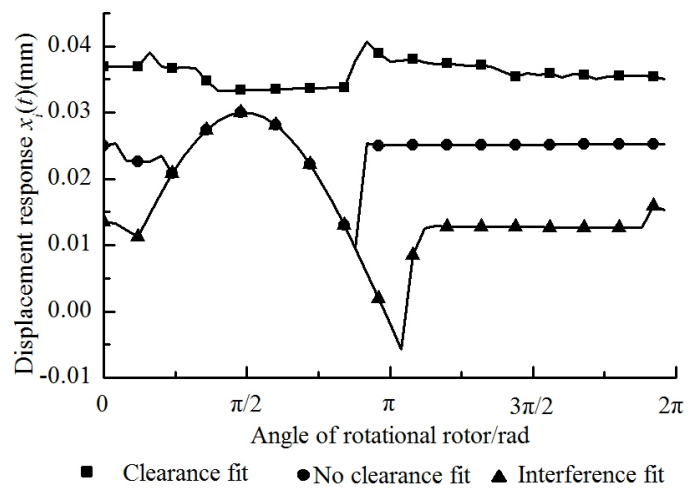

Fig.2 Effect of assembly condition on displacement response of finger stick



Fig.3 Effect of assembly condition on leakage gap of finger stick

Effect of assembly condition on contact pressure is shown in Fig.4. It is shown that, finger stick and the rotor is out of contact in the whole cycle when clearance fit. That indicates when clearance fit the following characteristic of finger stick is weakened. The contact pressure between finger stick and the rotor is maximum when interference fit, then the contact time is advance and the time of out contact is delay. That means when interference fit the wear of finger seal is obvious, and that makes the life of finger seal become short.

Average leakage gap and leakage rate under different assembly condition of 2.5 dimension $\mathrm{C} / \mathrm{C}$ composite finger seal is shown in Table 1. Corresponding to Fig.3, in accordance with clearance fit, no 
clearance fit and interference fit, the average leakage gap and leakage rate would be decreased.

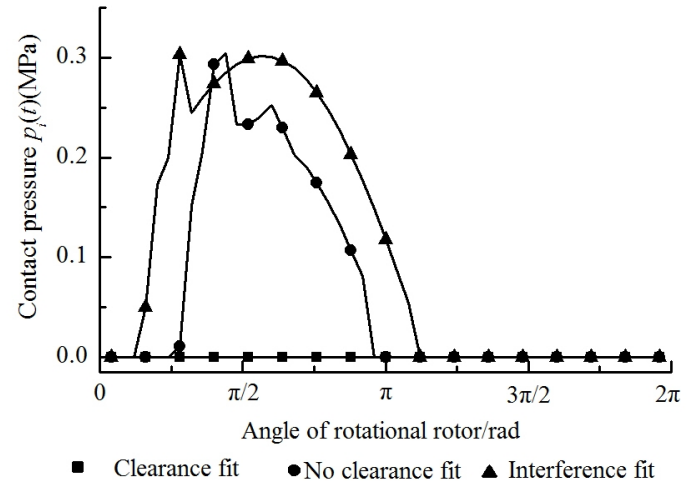

Fig.4 Effect of assembly condition on contact pressure

Table 1 Average leakage gap and leakage rate of different assembly condition

\begin{tabular}{ccc}
\hline Assembly condition & Average leakage gap $\bar{h}(\mathrm{~mm})$ & $\begin{array}{c}\text { Leakage rate } Q \\
(\mathrm{~g} / \mathrm{s})\end{array}$ \\
\hline Clearance fit & 0.0300 & 15.05 \\
No clearance fit & 0.0232 & 6.89 \\
Interference fit & 0.0157 & 2.12 \\
\hline
\end{tabular}

\section{Conclusions}

It is shown that the assembly condition has decisive influence on dynamic performance of finger seal in the paper. In accordance with clearance fit, no clearance fit and interference fit, the leakage gap of finger stick and the rotor would be decreased, while the contact pressure would be increased. It is reflected that the leakage and wear are two contradictory performance indexes of finger seal.

\section{Acknowledgement}

The study was supported by the Natural Science Foundation of Shaanxi Province (2014JM7266) and the National Natural Science Foundation of China (51305343).

\section{References}

[1] DU Kaibing, LI Yongjian, SUO Shuangfu, et al. Dynamic leakage analysis of noncontacting finger seals based on dynamic model[J], Journal of Engineering for Gas Turbines and Power, 2015,137(9):1-7.

[2] Proctor M P, Arun K, Delgado I R. High-speed, high-temperature finger seal test result[J]. Journal of Propulsion and Power, 2004,20(2):312-318.

[3] Arora G K. Dual pressure balanced non-contacting finger seal[P]. USA, US6364316 2002-04-02.

[4] Arora G K, Proctor M P, Steinetz B M, et al. Pressure balanced, low hysteresis, finger seal test results[R]. AIAA Paper 1999-2686, 1999.

[5] Gibson N E, Takeuchi D, Hynes T, et al. Second generation air-to-air mechanical seal design and performance[R]. AIAA Paper 2011-5636, 2011.

[6] CHEN Guoding, LU Fei, YU Qiangpeng, et al. Dynamic analysis of finger seal using equivalent model based on distributed mass method[J]. Journal of Mechanical Engineering Science, 2014,288(16):2992-3005.

[7] CHEN Guoding, WANG Li'na, YU Qiangpeng, et al. Dynamic analysis of C/C composite finger seal[J]. Chinese Journal of Aeronautics, 2014,27(3):745-758. 
[8] Marie H. A study of non-contacting passive-adaptive turbine finger seal performance[D]. Akron: The University of Akron, 2005. 